\title{
Strumaepidemiologie
}

\section{Schilddrüsenvolumina bei deutschen und schwedischen Schulkindern*}

\author{
R. Gutekunst, H. Smolarek, W. Wächter und P. C. Scriba
}

Klinik für Innere Medizin (Direktor: Prof. Dr. P. C. Scriba) der Medizinischen Hochschule Lübeck

Bei 13jährigen Schulkindern $(\mathrm{n}=2244)$ in 23 Orten der Bundesrepublik sowie bei 224 Schulkindern im ausreichend jodversorgten Stockholm wurde das Schilddrüsenvolumen sonographisch bestimmt. Dabei ergab sich, daß die Schilddrüsengröße von Norden nach Süden zunimmt. Deutliche Endemienester gibt es in den mittleren Regionen der Bundesrepublik. Als mittleres Schilddrüsenvolumen wurden bei den schwedischen Kindern 4,2 ml, bei den deutschen Kindern 9,3 ml ermittelt. Mehr als ein Drittel der deutschen Kinder haben ein größeres Schilddrüsenvolumen als das größte in Schweden gemessene Volumen. Die Ergebnisse stützen die Notwendigkeit der Jodsalzprophylaxe für die ganze Bundesrepublik.

Die Bundesrepublik Deutschland ist, wie zahlreiche lokale und überregionale Untersuchungen belegen (10, 12, 15, 22, 23, 27-29, 37), ein Kropfendemiegebiet. Bisherige deutsche Daten basierten auf der Auswertung von Röntgenbildern des Thorax $(5,8,14,28)$, auf der Palpation des Halses $(10,15,27,38)$ und Messung der Jodausscheidung $(10,12,23,37)$. Wie kürzlich gezeigt werden konnte $(8,9,38)$, sind Röntgenbilder für epidemiologische Zwecke ungeeignet, da die Sensitivität mit $24,3 \%$ und die Spezifität mit $91,4 \%$ gering ist. Die Palpation ist eine gute Methode, die allerdings der individuellen Beurteilung und Erfahrung des Arztes unterliegt. Die Jodausscheidung ist ein indirekter Parameter für die zu erwartende Strumahäufigkeit $(3,4,7,13,19,20,23$, 31). Kritisch ist bei den bisher vorliegenden überregionalen Daten ferner die Auswahl der Untersuchten. Die Ergebnisse von Horster und Mitarbeitern (15) beruhen auf Untersuchungen von Rekruten; Kinder, Frauen und Männer oberhalb des Musterungsalters wurden nicht erfaßt. Bisher gibt es schließlich in Deutschland keine Normalwerte für das Schilddrüsenvolumen (23), da alle, auch die zum Teil auswärts von Pathologen $(6,7,39)$ erhobenen Daten in Jodmangelgebieten erarbeitet wurden.

Unser Ziel war es, aktuelle und verläßlichere Daten über die Strumahäufigkeit zu erhalten. Wir haben daher

Dtsch. med. Wschr. 110 (1985), 50-54

(C) 1985 Georg Thieme Verlag Stuttgart · New York
Epidemiology of goitre. IV. Thyroid volumes in German and Swedish school-children

Thyroid volume was determined by ultrasound in 13 -year-old school-children $(n=2244)$ from 23 places in the Federal Republic of Germany and in 224 schoolchildren from Stockholm (where there is adequate iodine supply). The results show that thyroid size gradually increases from north to south. There are clear endemic nests in the central region of the Federal Republic of Germany. Mean thyroid volume in Swedish children was $4.2 \mathrm{ml}$, in German children $9.3 \mathrm{ml}$. More than one third of German children have a larger thyroid volume than the largest volume measured in Swedish children. The results support the need for iodine-salt prophylaxis for the entire German Federal Republic.

im Jodmangelgebiet Deutschland und im ausreichend jodversorgten Schweden als einen ersten Schritt entsprechend der Forderung der Weltgesundheitsorganisation (4) - kommt der Kropf in der Gesamtbevölkerung endemisch vor, sollen zuerst Kinder getrennt untersucht werden - das Schilddrüsenvolumen bei 13jährigen Kindern sonographisch untersucht.

\section{Probanden und Methode}

Das Schilddrüsenvolumen 13jähriger Schulkinder ( $\mathrm{n}=2244$ ) wurde an verschiedenen Schultypen in 23 Städten der Bundesrepublik (von Nord nach Süd: Albersdorf $\mathrm{n}=55$, Lübeck $\mathrm{n}=98$, Cloppenburg $\mathrm{n}=65$, Braunschweig $\mathrm{n}=78$, Münster/Westfalen $\mathrm{n}=89$, Melsungen $\mathrm{n}=140$, Aachen $\mathrm{n}=51$, Weilburg $\mathrm{n}=292$, Mangerskirchen $n=94$, Darmstadt $n=122$, Bamberg $n=43$, Neunkirchen $\mathrm{n}=115$, Homburg $\mathrm{n}=68$, Saarbrücken $\mathrm{n}=40$, Heilbronn $n=115$, Grafenau $n=54$, Augsburg $n=126$, Achern $\mathrm{n}=64$, Reutlingen $\mathrm{n}=151$, Ingolstadt $\mathrm{n}=83$, Neustadt/Titisee $\mathrm{n}=112$, Ravensburg $\mathrm{n}=75$, Garmisch-Partenkirchen $\mathrm{n}=114$ ) und in Schweden (Stockholm $\mathrm{n}=224$ ) sonographisch bestimmt. Mit wenigen Ausnahmen konnte der entsprechende Altersjahrgang der jeweiligen Schule insgesamt erfaßt werden. Nur wenige Eltern verweigerten die selbstverständlich freiwillige Teilnahme.

Länge, Breite und Dicke beider Schilddrüsenlappen wurden mit einem tragbaren Sonographiegerät (Sonoline 1000, Siemens), Linearschallkopf $405 \mathrm{MHz}$, mit Wasservorlaufstrecke gemessen. Echoveränderungen wurden festgehalten und dem Hausarzt übermittelt. Das Volumen wurde nach der Formel Dicke $\times$ Breite $\times$ Länge $\times$ Korrekturfaktor $(0,479)(2)$ errechnet. Ferner wurden Größe,

\footnotetext{
* Mit Unterstützung der Freunde und Förderer der Medizinischen
} Hochschule Lübeck 
Tab. 1. Schilddrüsenvolumina von 13jährigen Schulkindern in 23 Orten der Bundesrepublik $(n=2244)$ und in Stockholm ( $n=224)$

\begin{tabular}{|c|c|c|c|c|c|c|c|c|c|c|}
\hline \multirow{2}{*}{ Ort } & \multicolumn{5}{|c|}{ Knaben } & \multicolumn{5}{|c|}{ Mädchen } \\
\hline & $n$ & $\bar{x}$ & s & $\tilde{x}$ & Spanne & $n$ & $\bar{x}$ & s & $\tilde{\mathrm{x}}$ & Spanne \\
\hline Albersdorf & 26 & 6,842 & 2,333 & 6,15 & $3,0-13,8$ & 29 & 9,068 & 3,891 & 8,6 & $3,4-18,0$ \\
\hline Lübeck & 29 & 6,005 & 3,194 & 4,4 & $2,6-15,4$ & 69 & 8,013 & 4,620 & 6,7 & $2,9-26,4$ \\
\hline Braunschweig & 46 & 7,543 & 3,260 & 6,95 & $2,4-15,9$ & 32 & 9,115 & 4,986 & 7,85 & $2,5-22,0$ \\
\hline Melsungen & 72 & 12,465 & 5,118 & 10,95 & $3,6-26,6$ & 68 & 14,485 & 7,001 & 12,7 & $4,7-42,4$ \\
\hline Münster & 62 & 7,274 & 2,597 & 6,75 & $3,6-14,8$ & 27 & 10,518 & 5,638 & 9,0 & $3,3-28,1$ \\
\hline Cloppenburg & 26 & 6,592 & 2,610 & 6,0 & $3,3-13,9$ & 39 & 7,338 & 3,354 & 6,8 & $1,8-15,5$ \\
\hline Aachen & 26 & 9,765 & 6,682 & 8,75 & $2,6-39,3$ & 25 & 9,507 & 4,854 & 9,0 & $3,0-22,1$ \\
\hline Darmstadt & 84 & 8,323 & 3,505 & 7,55 & $2,9-22,8$ & 38 & 10,368 & 6,611 & 8,15 & $3,1-33,6$ \\
\hline Weilburg & 144 & 9,872 & 4,909 & 9,0 & $2,9-29,6$ & 148 & 12,258 & 6,610 & 10,15 & $2,7-40,9$ \\
\hline Mengerskirchen & 40 & 10,089 & 4,340 & 9,65 & $4,0-27,7$ & 54 & 11,629 & 4,576 & 10,95 & $3,8-26,2$ \\
\hline Saarbrücken & 26 & 7,719 & 4,522 & 6,5 & $1,5-19,4$ & 14 & 9,778 & 4,199 & 8,45 & $3,9-19,7$ \\
\hline Homburg & 24 & 7,724 & 4,631 & 5,95 & $2,6-18,7$ & 44 & 9,740 & 6,708 & 8,0 & $1,4-35,3$ \\
\hline Neunkirchen & 71 & 7,745 & 5,476 & 6,8 & $2,3-40,7$ & 44 & 8,752 & 4,882 & 7,4 & $3,1-25,8$ \\
\hline Heilbronn & 45 & 6,557 & 3,100 & 6,0 & $1,8-13,3$ & 70 & 8,764 & 4,469 & 7,8 & $2,3-22,3$ \\
\hline Reutlingen & 81 & 7,513 & 4,519 & 6,6 & $2,3-28,8$ & 70 & 9,031 & 5,213 & 7,8 & $2,0-26,7$ \\
\hline Achern & 39 & 6,384 & 3,532 & 5,7 & $2,0-18,1$ & 25 & 6,775 & 2,768 & 5,9 & $1,9-11,2$ \\
\hline Neustadt-Titisee & 65 & 6,527 & 3,695 & 5,7 & $1,1-20,3$ & 47 & 8,159 & 6,756 & 7,0 & $2,2-47,7$ \\
\hline Ravensburg & 47 & 5,919 & 2,744 & 5,4 & $1,9-13,3$ & 28 & 7,192 & 3,405 & 6,6 & $1,6-16,7$ \\
\hline Ingolstadt & 44 & 10,743 & 5,714 & 9,5 & $2,1-26,0$ & 39 & 12,612 & 7,391 & 10,7 & $4,9-41,4$ \\
\hline Garmisch & - & - & - & - & - & 114 & 12,612 & 7,391 & 9,0 & $1,9-23,3$ \\
\hline Grafenau & 23 & 12,034 & 5,716 & 11,3 & $5,0-27,1$ & 31 & 14,406 & 7,503 & 12,5 & $5,5-31,4$ \\
\hline Bamberg & 35 & 11,271 & 4,441 & 9,8 & $5,2-25,1$ & 8 & 13,062 & 6,275 & 13,2 & $3,6-22,4$ \\
\hline Augsburg & - & - & - & - & - & 126 & 8,958 & 4,214 & 8,1 & $2,4-29,7$ \\
\hline Stockholm & 110 & 4,118 & 1,652 & 3,9 & $1,2-9,4$ & 114 & 4,250 & 1,722 & 4,0 & $1,4-11,5$ \\
\hline
\end{tabular}

Gewicht, Geburtsort, Wohnortwechsel, Schilddrüsenerkrankungen in der Familie, Medikamenteneinnahme und der Gebrauch von jodiertem Speisesalz erfragt.

\section{Ergebnisse}

1. Schilddrüsenvolumina. Die Tabelle 1 zeigt die Mittelwerte, Standardabweichungen, Mediane und Spannen der Schilddrüsenvolumina für die einzelnen Städte, aufgeteilt in Mädchen und Jungen. Das mittlere Schilddrüsenvolumen in der Bundesrepublik beträgt für Jungen 8,5 $\pm 4,7 \mathrm{ml}$ (Spanne 1,1-40,7 ml), für Mädchen 10,1 \pm 5,7 $\mathrm{ml}$ (Spanne 1,4-47,7 $\mathrm{ml}$ ) und gemeinsam für beide Geschlechter 9,3 $\pm 5,3 \mathrm{ml}$, während der Gesamtmittelwert in Stockholm 4,2 ml ist.

2. Volumenverteilung. In der Abbildung 1 sind die mittleren Schilddrüsenvolumina für Knaben und Mädchen in geographischer Verteilung dargestellt. Die Abbildung 2 zeigt für jeden Ort den Anteil in Prozent der Kinder, bei denen ein größeres Schilddrüsenvolumen gefunden wurde als $7,4 \mathrm{ml}$ für Knaben und $7,7 \mathrm{ml}$ für Mädchen. Diese Volumengrenzen entsprechen den schwedischen Mittelwerten mit 2 Standardabweichungen. Im Mittel hatten $49 \%$ der Jungen und $59 \%$ der
Mädchen in der Bundesrepublik ein Schilddrüsenvolumen, das über dem Normalbereich $(\bar{x} \pm 2 s)$ der schwedischen Schulkinder lag. Die Abbildung 3 veranschaulicht vergleichend die Häufigkeitsverteilung der Schilddrüsenvolumina in beiden Ländern. Beide Verteilungen sind annähernd glockenförmig, wobei die deutschen Kurven im Vergleich zu den schwedischen nach rechts verschoben sind.

3. Echostrukturveränderungen. Insgesamt fanden sich bei 18 deutschen Kindern $(0,8 \%)$ Echostrukturveränderungen. Fünf Kinder $(0,2 \%)$ hatten diffus echoverarmte und drei $(0,15 \%)$ diffus echoreiche Schallmuster. Bei sechs Kindern $(0,3 \%)$ fand sich eine Zyste, bei drei Kindern $(0,15 \%)$ ein Knoten, und bei einem Kind $(0,04 \%)$ bestanden Kalkablagerungen. Bei den schwedischen Kindern kamen keine Echostrukturabweichungen vor.

4. Anamnestische Angaben. Alle Kinder wohnten länger als ein Jahr, 94\% länger als 5 Jahre am Schulort oder in der näheren Umgebung. $80 \%$ wurden innerhalb von $100 \mathrm{~km}$ vom Schulort entfernt geboren. 13,6\% der Kinder gaben Schilddrüsenerkrankungen in der Familie an; 


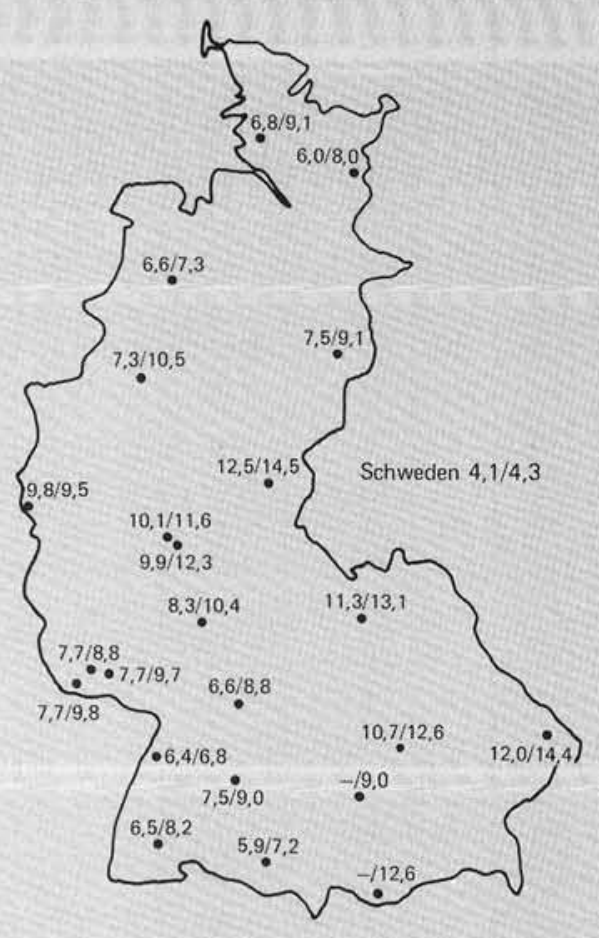

Abb. 1. Schilddrüsenvolumina $(\mathrm{ml})$ bei 13jährigen Schulkindern $(n=2244)$ in der Bundesrepublik. Die erste Zahl ist der Mittelwert für Jungen, die zweite der Mittelwert für Mädchen.

30,2\% konnten keine Angaben machen. Die Mädchen, in deren Familien Schilddrüsenerkrankungen vorkamen, hatten ein größeres mittleres Schilddrüsenvolumen $(11,4$ $\pm 6,4 \mathrm{ml}$ ) als diejenigen, von deren Familienangehörigen keine Schilddrüsenerkrankungen bekannt waren $(10,0$ $\pm 5,6 \mathrm{ml}$ ). Bei den Jungen fand sich kein Unterschied. Die Fragen nach Medikamenteneinnahme und Jodsalzgebrauch wurden sehr unsicher beantwortet. Bei $15,4 \%$ war Jodsalz angeblich eingeführt, während $83,6 \%$ der Kinder entweder keine Angaben machen konnten oder kein Jodsalz verwenden. Zwischen den Schilddrüsenvolumina beider Gruppen bestand kein Unterschied. Allerdings müssen die anamnestischen Angaben der Kinder angezweifelt werden, weshalb Rückschlüsse kaum zulässig erscheinen.

5. Korrelation von Schilddrüsenvolumen, Körpergewicht, Körpergröße und Körperoberfläche. a) Schilddrüsenvolumen und Körpergewicht. Zwischen Schilddrüsenvolumen und Körpergewicht bestand im Vergleich zur Körpergröße bzw. Körperoberfläche die beste Korrelation. Allerdings war der Korrelationskoeffizient für Mädchen in Deutschland $\mathrm{r}=0,364(P<0,001)$ und für Schwedinnen $\mathrm{r}=0,18(P<0,05)$, für deutsche Jungen $\mathrm{r}=0,434(P<0,001)$ und für schwedische Jungen $\mathrm{r}=0,491(P<0,001)$ nur schwach.

b) Schilddrüsenvolumen und Körpergröße. Die Korrelation zwischen Schilddrüsenvolumen und Körper-

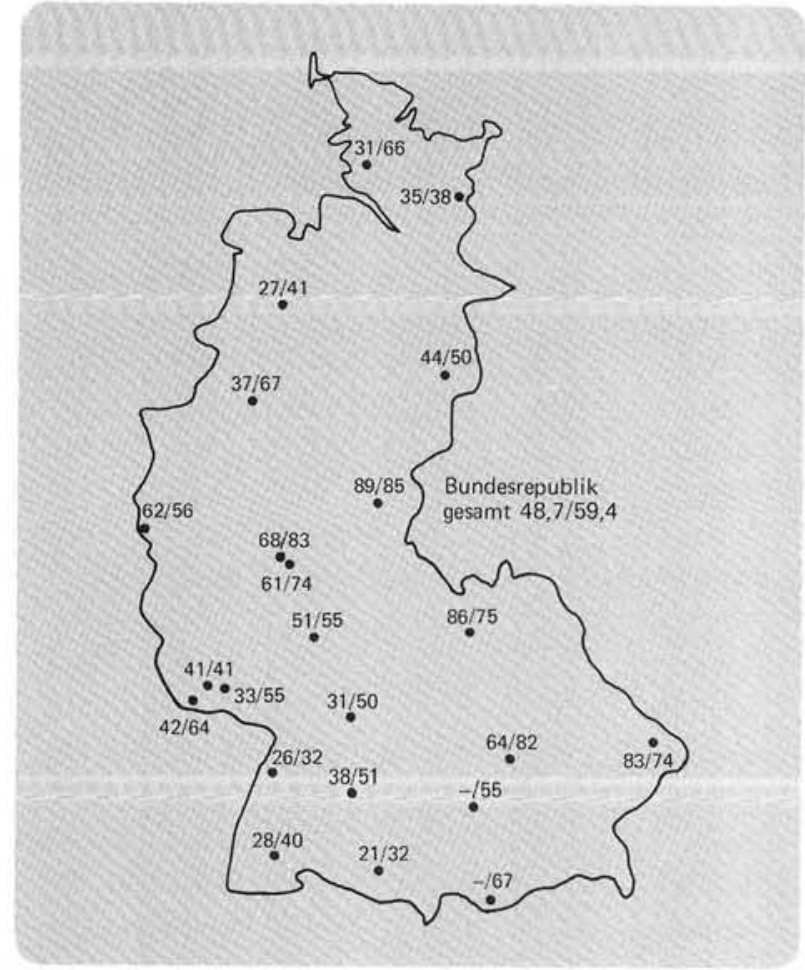

Abb. 2. Anteil der Schilddrüsenvolumina bei 13jährigen Schulkindern $(n=2244)$ in der Bundesrepublik in Prozent, der das in Schweden festgestellte mittlere Schilddrüsenvolumen $(n=224)$ plus zwei Standardabweichungen übersteigt. Vor dem Schrägstrich stehen die Werte für die Jungen, dahinter für die Mädchen.

größe ist schlechter als die zwischen Körpergewicht und Schilddrüsenvolumen: deutsche Mädchen $\mathrm{r}=0,236$ $(P<0,001)$, schwedische Mädchen $0,155(P<0,05)$, deutsche Jungen $r=0,259(P<0,001)$, schwedische Jungen $r=0,483(P<0,001)$.

c) Schilddrüsenvolumen und Körperoberfläche: deutsche Mädchen $\mathrm{r}=0,358(P<0,001)$, schwedische Mädchen $\mathrm{r}=0,187(P<0,05)$, deutsche Jungen $r=0,396(P<0,001)$, schwedische Jungen $r=0,117$ $(P<0,05)$.

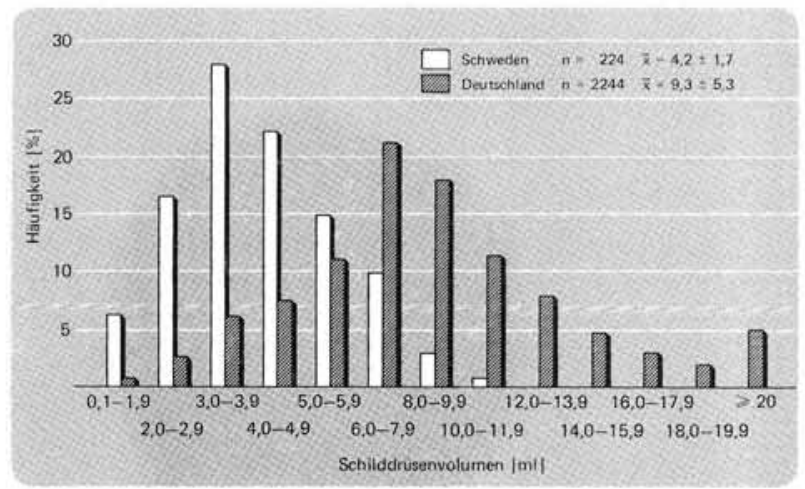

Abb. 3. Häufigkeitsverteilung der Schilddrüsenvolumina für Jungen und Mädchen gemeinsam. 


\section{Diskussion}

Die Palpation war bei bisherigen epidemiologischen Studien über die Strumaprävalenz bei Kindern die einzige Methode $(4,10,27,37)$. Gerade bei 13jährigen Kindern, die am Anfang oder bereits in der Pubertät sind, kann besonders bei schlankem und adipösem Hals die Schilddrüsengröße oder Struma, vor allem des Stadiums I nach WHO, palpatorisch über- oder unterschätzt werden $(4$, 22). Unterschiede der individuellen Beurteilung bei mehreren Untersuchern kommen hinzu, zumal es für Kinder bisher kaum überregionale Studien gibt. Die sonographische Volumetrie versprach verläßlichere, reproduzierbare Daten $(1,2,9,11,16-18,21,22,38)$.

Auf die endlosen organisatorischen, bürokratischen, juristischen, politischen und administrativen Schwierigkeiten, die heute bei der Planung und Durchführung einer solchen Studie entstehen, soll hier nur nebenbei hingewiesen werden.

Verschiedene Untersucher $(10,23,27,32,37)$ hatten schon vor Jahren auf das Joddefizit bei Kindern in der Bundesrepublik hingewiesen. Heidemann und Mitarbeiter (12) konnten kürzlich entsprechende Daten auch für Neugeborene vorlegen. In Schweden wurde in den dreißiger und vierziger Jahren stufenweise zunächst mit $10 \mathrm{mg}$, dann mit $20 \mathrm{mg}$ Jod pro kg Salz die Jodsalzprophylaxe eingeführt. Seit 1966 wird dem üblichen Tafelsalz, dem Salz zur Babynahrungsherstellung und dem Salz für die Tiernahrung $50 \mathrm{mg}$ Jod pro kg zugesetzt ( 35 ; Becker, W. [Uppsala]: persönliche Mitteilung). Seither ist der Kropf in Schweden so gut wie ausgerottet. Entsprechend liegt die Jodausscheidung in Schweden vier- bis zehnmal höher als in der Bundesrepublik (12, 32).

Übereinstimmend mit Horster und Mitarbeitern (15) sowie Habermann und Mitarbeitern (10) lassen unsere Daten ebenfalls ein Süd-Nord-Gefälle des Schilddrüsenvolumens erkennen. Allerdings ist die Häufigkeit der vergrößerten Schilddrüsen im Norden deutlich höher, als nach der Arbeit von Horster und Mitarbeitern (15) erwartet wurde. Übereinstimmend mit eigenen Ergebnissen bei Erwachsenen in Ostholstein $(8,9)$ und mit Erhebungen von Schemmel und Greven (27) zur Strumaprävalenz bei Schulkindern in Dithmarschen muß auch Schleswig-Holstein als Kropfendemiegebiet angesehen werden. Ferner gibt es im Westerwald, in Nordhessen und im bayerischen Wald besonders deutliche lokale Endemienester.

Überraschend waren die kleinen Schilddrüsenvolumina in Ravensburg. Recherchen ergaben, daß bis vor etwa einem Jahr in den dem Gesundheitsamt Ravensburg zugehörigen Volksschulen einmal wöchentlich eine Tablette mit 3 mg Kaliumjodid verteilt wurde. Wegen juristischer Unsicherheiten wurde die Prophylaxe durch das Sozialministerium (zunächst?) wieder ausgesetzt.

Die Jungen in der Bundesrepublik hatten ein kleineres Schilddrüsenvolumen als die Mädchen, wie es für Erwachsene schon lange bekannt ist $(1,11,16,21,22$, 25, 39). Die schwedischen Schilddrüsenvolumina waren erwartungsgemäß erheblich kleiner. Die schwedischen Mädchen hatten nur geringfügig größere Schilddrüsen als die schwedischen Jungen.

Die Volumenverteilung in der Bundesrepublik und in Schweden ist glockenförmig. In keinem Verteilungsmuster, auch nicht bei getrennter Aufzeichnung nach Geschlecht, fand sich ein zweiter Häufigkeitsgipfel, den man einem speziellen pathogenetischen Faktor (Heterogenität) hätte zuordnen können. Die Volumenhäufigkeitsverteilung ist in der Bundesrepublik im Vergleich zu Schweden lediglich nach rechts verschoben. Das bedeutet, alle Kinder werden von der Jodversorgung gleichermaßen, aber mit unterschiedlicher Intensität beeinflußt. Das macht es schwer, wenn nicht unmöglich, einerseits einen Toleranzbereich für das normale Schilddrüsenvolumen zu definieren und andererseits eine Aussage über die wahre Strumaprävalenz zu machen. Die Schilddrüsengröße der deutschen Jugendlichen übertrifft aber in mehr als der Hälfte der Fälle den in Schweden ermittelten Normalbereich $(\bar{x} \pm 2$ s) und ist somit neutral als $»$ vergrößert « zu bezeichnen; mehr als ein Drittel der deutschen Werte liegt über dem höchsten in Stockholm gemessenen Schilddrüsenvolumen.

Vergleicht man die von uns sonographisch ermittelten Volumina mit den lokalen Angaben über die Strumaprävalenz aufgrund von Palpationsbefunden in Dithmarschen (27) und in Göttingen (37), so kommt man zu einer recht guten Korrelation. Mit Sicherheit läßt sich schließen, daß bereits die kindlichen Schilddrüsen als Folge des Jodmangels unter einem erheblichen Kompensationsdruck stehen, dem sie mit Hyperplasie und Hypertrophie begegnen, dem Beginnn der Organerkrankung Struma.

Wie Olbricht und Mitarbeiter (22) fanden wir nur eine schwach positive Korrelation zwischen Körpergröße, Körpergewicht, Körperoberfläche und Schilddrüsenvolumen. Am deutlichsten war der Zusammenhang zwischen Körpergewicht und Schilddrüsenvolumen. Zu gleichen Ergebnissen kamen auch Hegedüs und Mitarbeiter (11) für Erwachsene.

Unsere Ergebnisse belegen erneut die Wirksamkeit der Jodsalzprophylaxe. Dementsprechend fordern wir alle Verantwortlichen - Ärzte, Gesundheitsämter, Gesundheitspolitiker und Medien - auf, Wege für eine erfolgreiche Jodprophylaxe zu finden $(4,13,19,20,23,26$, 29-31, 33, 34). Wie Pfannenstiel (24) ausgerechnet hat, könnte damit mittelfristig ein wesentlicher Beitrag zur Kostendämpfung im Gesundheitswesen in der Höhe von mehr als einer halben Milliarde DM jährlich geleistet werden.

\section{Literatur}

(1) Brown, M. C., R. Spencer: Thyroid gland volume estimated by use of ultrasound in addition to scintigraphy. Acta radiol. (Stockh.) 17 (1978), 337.

(2) Brunn, J., U. Block, G. Ruf, J. Bos, W P. Kunze, P. C. Scriba: Volumetrie der Schilddrüsenlappen mittels Real-time-So(1981), 1338.

(3) Bürgi, H.: Pathophysiologie des euthyreoten Kropfes. Therapiewochc 30 (1980), 962 .

(4) Dunn, J. T., G. A. Medeiros-Neto (Ed.): Endemic Goiter and Cretinism: nographie. Dtsch. med. Wschr. 106 
Continuing Threats to World Health. Pan American Health Organization, Scientific Publication No. 292 (World Health Organization: Washington D. C. 1974), 1.

(5) Finger, E., J. Dabels, G. Schünemann, M. Krüger: Strumareihenuntersuchung durch Volksröntgenaktion. Z. Erkrank. Atm.-Org. 158 (1982), 309.

(6) Fischer, H. E.: Das Gewichtswachstum der menschlichen Schilddrüse im saarländisch-pfälzischen Raum. Inauguraldissertation, Universität des Saarlandes, Homburg, 1980.

(7) Gerber, D.: Schilddrüsengewichte und Jodsalzprophylaxe. Schweiz. med.

Wschr. 110 (1980), 2010.

(8) Gutekunst, R., C. Schütte, B. Windler, P. C. Scriba: Kritik der Strumaepidemiologie. Il. Altersabhängigkeit. Dtsch. med. Wschr. 108 (1983), 1016.

(9) Gutekunst, R., K. Groth, B. Windler, P. C. Scriba: Kritik der Strumaepidemiologie. III. Vergleich von Röntgen-Thoraxaufnahmen, Palpation und sonographischer Volumetric. In Schleusener, H., et al. (Hrsg.): Schilddrüse 1983 (Thieme: Stuttgart 1984), im Druck.

(10) Habermann, J., H. G. Heinze, K. Horn, R. Kantlehner, I. Marschner, J. Neumann, P. C. Scriba: Alimentärer Jodmangel in der Bundesrepublik Deutschland. Dtsch. med. Wschr. 100 (1975), 1937.

(11) Hegedüs, L., H. Perrild, L. R. Poulsen, J. R. Andersen, B. Holm, P. Schnohr, G. Jensen, J. M. Hansen: The determination of thyroid volume by ultrasound and its relationship to body weight, age and sex in normal subjects. J. clin. Endocr. 56 (1983), 260.

(12) Heidemann, P. H., P. Stubbe, K. v, Reuss, P. Schürnbrand, A. Larson, W. y. Petrykowski: Jodausscheidung und alimentäre Jodversorgung bei Neugeborenen in Jodmangelgebieten der Bundesre publik. Dtsch. med. Wschr. 109 (1984), 773.

(13) Hetzel, S.: lodine deficiency disorders (IDD) and their eradication. Lance 1983/II, 1126
(14) Himanka, E., L.-G. Larsson: Estimation of thyroid volume. Acta radiol. (Stockh.) 43 (1955), 125.

(15) Horster, F. A., G. Klusmann, W. Wildmeister: Der Kropf: eine endemische Krankheit in der Bundesrepublik? Dtsch. med. Wschr. 100 (1975), 8.

(16) Igl, W., U. Seiderer, U. Fink, J. Lissner: Quantitative Volumenbestimmung der Schilddrüse mit Hilfe der Sonographie. Nuc Compact 11 (1980), 11.

(17) Igl, W., U. Fink, B. Leisner, A. Gebauer: Die Ultraschalldiagnostik der Struma. Therapiewoche 31 (1981), 1609.

(18) Igl, W., F. Lukas, B. Leisner, U. Fink, M. Seiderer, C. R. Pickardt, J. Lissner: Sonographische Volumenbestimmung der Schilddrüse - Vergleich mit andere Methoden. Nucl.-Med. 20 (1981), 64.

(19) Lamberg, B. A., M. Haikonen, M. Mäkelä, A. Jukkara, E. Axelson, M. G. Welin: Further decrease in thyroidal uptake and disappearance of endemic goite in children after 30 years of iodine prophylaxis in the East of Finland. Acta en docr. (Kbh.) 98 (1981), 205.

(20) Lamberg, B.-A., M. Haikonen, M. Mäkelä, A. Jukkara: Wirksamkeit der Jodprophylaxe in Ostfinnland. In Scriba, P. C., K.-H. Rudorff, B. Weinheimer (Hrsg.): Schilddrüse 1981 (Thieme: Stuttgart-New York 1982), 248.

(21) Meissner, J., H. Weiss: Ergebnisse sonographisch-planimetrischer Messungen zur Volumenbestimmung der Schilddrüse. Dtsch. Ges. Ultraschall, Kongreßband (Wien 1977), 270.

(22) Olbricht, T., T. Schmitka, U. Mel. linghoff, G. Benker, D. Reinwein: Sonographische Bestimmung von Schilddrüsenvolumina bei Schilddrüsengesunden. Dtsch. med. W/schr. 108 (1983), 1355

(23) Pfannenstiel, P., F. A. Horster: Jodmangel in der Bundesrepublik Deutschland. Dtsch. med. Wschr. 107 (1982), 867.

(24) Pfannenstiel, P.: Direct and indirect costs evoked by continuous iodine deficiency. Serono Symposium: Thyroid Dis- orders Associated with Iodine Deficiency and Excess. Freiburg 1984 (im Druck).

(25) Rasmussen, N., L. Hjorth: Determination of the thyroid volume by ultrasonic scanning. J. clin. Ultrasound 2 (1974), 143.

(26) Reiser, H., K. J. Schmidt, G. Rothenbuchner: Derzeitiger Stand der Jodsalzprophylaxe in der Bundesrepublik Deutschland. Therapiewoche 33 (1983), 5928.

(27) Schemmel, K., H. Greven: Strumahäufigkeit und Jodurie bei 13-15jährigen im Dithmarschen. In Schleusener, H., et al. (Hrsg.): Schilddrüse 1983 (Thieme: Stuttgart-New York 1984), im Druck. (28) Schoknecht, G., G. Barich: Bestimmung der Häufigkeitsverteilung von Strumen mit Röntgenschirmbildaufnahmen bei Filteruntersuchungen. Dtsch. med. Wschr. 99 (1974), 1860.

(29) Scriba, P. C., J. Kracht, E. Klein: Endemische Struma-Jodsalzprophylaxe. Dtsch. med. Wschr. 100 (1975), 1350. (30) Scriba, P. C.: Strumaprophylaxe durch jodiertes Speisesalz. Dtsch. Ärz tebl. 74 (1977), 1955.

(31) Scriba, P. C., C. R. Pickardt: Strumaprophylaxe. Intern. Welt 11 (1980), 409.

(32) Scriba, P. C.: Epidemiology of iodine deficiency in Europe. Serono-Sympo-

Dr. R. Gutekunst, cand. med. H. Smolarek, Dr. W. Wächter, Prof. Dr. P. C. Scriba

Klinik für Innere Medizin

Medizinische Hochschule

2400 Lübeck 1, Ratzeburger Allee 160 sium: Thyroid Disorders Associated with lodine Deficiency and Excess. Freiburg 1984 (im Druck).

(33) Scriba, P. C.: Strumaprophylaxe wann endlich? Dtsch, med. W/schr. 107 (1982), 843.

(34) Sechste Verordnung zur Änderung der Diätverordnung vom 7. Juli 1981, Bundesgesetzblatt, Jahrgang 1981, Teil I, Seite $613, \$ 3, \$ 10$ und 11 a, $\$ 24,26$ 27 a (2).

(35) Statens Livsmedelsverks Kungörelse om Berikningsmedel i Livsmedel. 4 februari 1983, SLV FS 1983: 2, \$9 och bilaga 3 .

(36) Steck, A., B. Steck, M. P. König, H. Studer: Auswirkungen einer verbesserten Jodprophylaxe auf Kropfendemie und Jodstoffwechsel. Schweiz. med. Wschr. 102 (1972), 829.

(37) Stubbe, P., P. Heidemann: Struma neonatorum - blande Struma im Kindes alter. Dtsch. Ärztebl. 80 (1983), 40.

(38) Waller, D. E., R. Gutekunst, D. Brossmann, P. C. Scriba: Kritik der Strumaepidemiologie. 1. Strumagröße. Dtsch. med. Wschr. 108 (1983), 1014

(39) Wegelin, C.: Handbuch der spezielen pathologischen Anatomie und Histologie. Bd, VIII. Drüsen mit innerer Sekretion (Springer: Berlin 1926). 